Z Rheumatol 2020 $79: 865-872$

https://doi.org/10.1007/s00393-020-00852-w

Online publiziert: 26 . August 2020

(C) Der/die Autor(en) 2020

\section{Redaktion}

H.-I. Huppertz, Bremen

U. Müller-Ladner, Bad Nauheim

\author{
T. Welzel ${ }^{1,2} \cdot$ A. Wörner ${ }^{1} \cdot$ U. Heininger ${ }^{3,4}$ \\ ' Pädiatrische Rheumatologie, Universitäts-Kinderspital beider Basel (UKBB), Universität Basel, Basel, \\ Schweiz \\ ${ }^{2}$ Pädiatrische Pharmakologie, Universitäts-Kinderspital beider Basel (UKBB), Universität Basel, Basel, \\ Schweiz \\ ${ }^{3}$ Pädiatrische Infektiologie, Universitäts-Kinderspital beider Basel (UKBB), Universität Basel, Basel, Schweiz \\ ${ }^{4}$ Medizinische Fakultät, Universität Basel, Basel, Schweiz
}

\title{
Reiseimpfungen bei rheumatischen Erkrankungen
}

\section{Besonderheiten bei Kindern und Erwachsenen}

Rheumatische Erkrankungen (RE) betreffen Kinder und Erwachsene. Patienten mit RE haben ein erhöhtes Risiko, an bestimmten Infektionen zu erkranken, wobei das Infektionsrisiko von der Grunderkrankung und der individuellen immunsuppressiven Therapie (IT) abhängt $[16,20]$. Auch haben immunsupprimierte Patienten ein erhöhtes Risiko, während und nach Reisen an allgemeinen und reisespezifischen Infektionen schwerer zu erkranken, als Immunkompetente [7, 13]. Dieser Beitrag bietet Hilfestellung für die reisemedizinische Impfberatung bei Patienten mit RE. Die Fachinformationen der Impfstoffe und der IT müssen bei der individuellen Patientenberatung zusätzlich beachtet werden.

\section{Bedeutung von Impfungen bei rheumatischen Erkrankungen}

Patienten mit RE haben ein erhöhtes Infektionsrisiko für virale, bakterielle, fungale oder parasitäre Infektionen, was von der Grunderkrankung und der immunsuppressiven Therapie (IT) abhängt $[16,20]$. Auch verlaufen impfpräventable Krankheiten bei Patienten mit RE und IT schwerer und komplikationsträchtiger als bei Immunkompetenten $[13,29]$. Da sich die Lebensqualität von Patienten mit RE durch die IT deutlich gebessert hat, steigt die Nachfrage nach Fernreisen, und viele scheinen mittler- weile ein ähnliches Reiseverhalten wie immunkompetente Reisende zu zeigen [17]. Eine Untersuchung deutet dabei an, dass immunsupprimierte Patienten oft unzureichend auf die Reise vorbereitet sind, selbst bei Reisen in Hochrisikogebiete [3]. Da immunsupprimierte Patienten ein erhöhtes Risiko haben, während und nach Reisen an allgemeinen und reisespezifischen Infektionen zu erkranken [7, 13], sind für sie die von der STIKO (Ständige Impfkommission) empfohlenen Standard- und Indikationsimpfungen sowie Reiseimpfungen von großer Bedeutung. Für Patienten mit autoinflammatorischen Erkrankungen gelten im Wesentlichen dieselben Anwendungshinweise wie für Patienten mit autoimmunologischen RE [9].

\section{Totimpfstoffe}

Totimpfstoffe enthalten inaktivierte Erreger oder immunogene Erregerbestandteile. Sie können bei Patienten mit RE mit und ohne IT sicher verabreicht werden $[11,12,14,21,25,29]$. Für einen optimalen Impferfolg sollte die Impfung 2, besser 4 Wochen vor IT abgeschlossen sein (Rituximab $\geq 4$ Wochen) [29]. Während der IT sollte bei stabiler Erkrankung und/oder möglichst geringer IT geimpft werden [4, 29].

Die Immunogenität der Impfung wird durch die Grunderkrankung und die IT beeinflusst und kann im Ver- gleich zu Gesunden reduziert sein [12, $21,29]$. Meist wird aber ein ausreichender Impfschutz erreicht, ausgenommen sind B- und/oder T-Zell-depletierende Antikörpertherapien [29]. Die Kontrolle des Impferfolgs durch Messung von spezifischen Antikörpern (AK) nach abgeschlossener Impfserie mit Erreichen bzw. Überschreiten definierter Schutzkorrelate kann sinnvoll sein [21].

\section{Lebendimpfstoffe}

Lebendimpfstoffe enthalten abgeschwächte (attenuierte), aber noch replikationsfähige Viren oder Bakterien. Bei Patienten mit RE ohne IT können Lebendimpfstoffe ohne zusätzliche (zu den allgemein in der Fachinformation erwähnten) Sicherheitsbedenken verabreicht werden; ein Mindestabstand von 4 Wochen vor ITBeginn sollte eingehalten werden [25, 29]. Unter IT sollten allgemein keine Lebendimpfungen verabreicht werden $[14,25,29]$. Abhängig vom Schweregrad der IT können jedoch nach individueller Risiko-Nutzen-Abwägung bestimmte Lebendimpfungen erwogen werden [14, 29].

\section{Reisen mit rheumatischen Erkrankungen}

Vor Reisen sollten Personen mit RE nach den üblichen reisemedizinischen Empfehlungen (z. B. Deutsche Gesellschaft für 


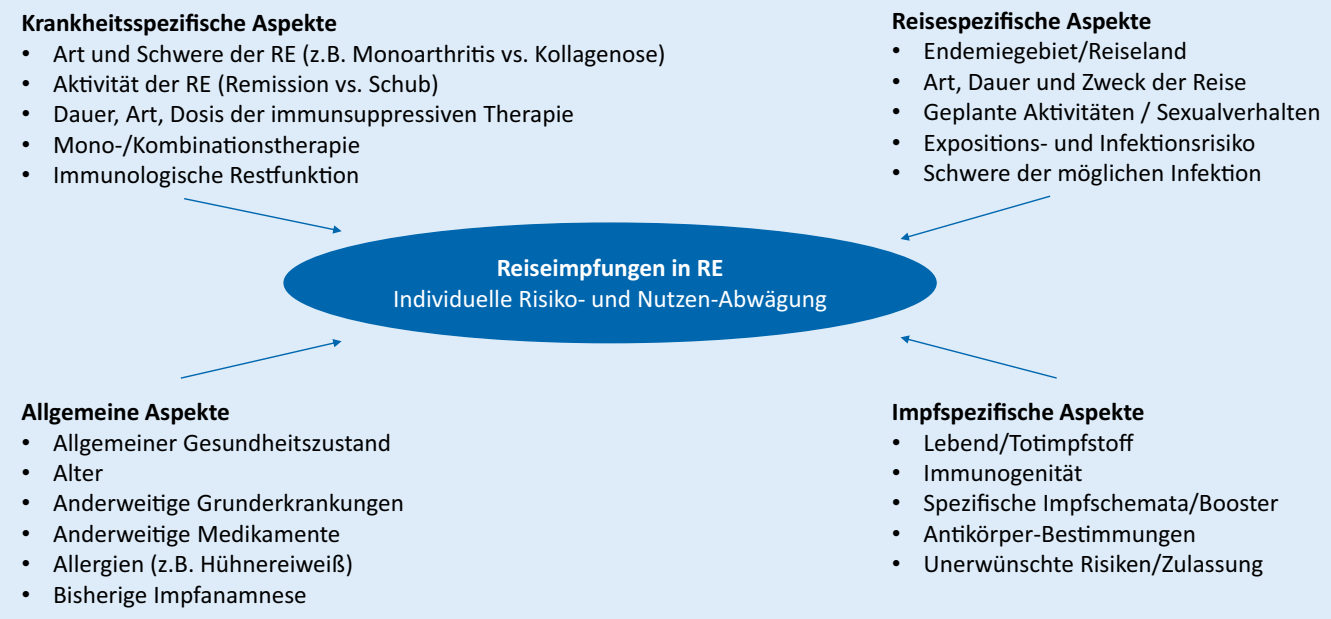

Reiseimpfungen in RE Individuelle Risiko- und Nutzen-Abwägung

$\longrightarrow$ Ind

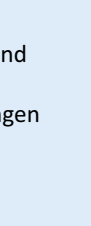

Reisespezifische Aspekte

- Endemiegebiet/Reiseland

- Art, Dauer und Zweck der Reise

- Geplante Aktivitäten / Sexualverhalten

- Expositions- und Infektionsrisiko

- Schwere der möglichen Infektion

\author{
Impfspezifische Aspekte \\ - Lebend/Totimpfstoff \\ - Immunogenität \\ - Spezifische Impfschemata/Booster \\ - Antikörper-Bestimmungen \\ - Unerwünschte Risiken/Zulassung
}

Abb. $1<$ Individuelle Risiko-Nutzen-Abwägung bei Reiseimpfungen bei Patienten mit rheumatischen Erkrankungen (RE) (angelehnt an $[10,19])$.
Tropenmedizin und Internationale Gesundheit, www.dtg.org) beraten werden. Neben der Impfberatung ist die Aufklärung über allgemeine Verhaltensmaßnahmen wie Hände- und Nahrungsmittelhygiene sowie Mückenschutz essenziell. Auch sollte der Reisende beraten werden, ob ein Reiserücktritt oder eine Reisemodifikation in Anbetracht des individuellen Risikos sinnvoll ist. Welche Reiseimpfungen indiziert sind, ergibt sich aus der möglichen Exposition gegenüber impfpräventablen Infektionen, der Schwere der möglichen Infektion und den Risiken, die mit der Reiseimpfung assoziiert sind [10]. In diesen Entscheidungsprozess sollte auch das individuelle Reiseverhalten des Patienten einbezogen werden [10]. Hierzu gehören zumindest Art, Dauer und Zweck der Reise, Unterkunft und Ernährung sowie geplante Aktivitäten vor Ort und das Sexualverhalten [10]. Bei Patienten mit RE müssen zusätzlich der allgemeine Gesundheitszustand, die Aktivität und die Schwere der RE sowie der Grad der IT beachtet werden. Dabei ist es auch wichtig, die Art des Impfstoffs (Tot-/Lebendimpfstoff) zu berücksichtigen (• Abb. 1).Zusätzlich muss geprüft werden, ob mögliche Interaktionen zwischen ggf. nötiger Chemoprophylaxe (z. B. bei Malaria) und IT vorliegen.

\section{Ausmaß der Immunsuppression}

Vor Impfung ist eine individuelle Einschätzung der aktuellen Immunkompetenz notwendig, die durch den allgemei- nen Gesundheitszustand, die Aktivität und Art der RE, Dosis und Art sowie Dauer der IT beeinflusst wird [29]. Einen Anhalt zur Einordnung der immunsuppressiven Wirkung bestimmter IT, basierend auf im Wesentlichen empirischen Daten und Expertenerfahrungen, fasst • Tab. 1 zusammen. Spezifische Tests können helfen, die immunologische Reaktionsfähigkeit des Patienten unter IT besser einzuordnen [26]. Folgende Bestimmungen können im Bedarfsfall vor Lebendimpfung gemäß empirischen Daten und Expertenmeinungen hilfreich sein und werden vorgeschlagen [26]:

- Lymphozytenzahl $\geq 1200 / \mu \mathrm{l}$ und/oder CD4(+)-T-Helferzellen $\geq 200 / \mu \mathrm{l}(\mathrm{ab}$ Alter 6 Jahre) bzw. $\geq 500 / \mu$ l (Alter 0 bis 5 Jahre), ergänzend für Patienten nach Rituximab-Therapie: B-Zellen sollten vor Lebendimpfung wieder im Altersnormbereich sein,

- Immunglobulin $\mathrm{G} \geq(5-) 7 \mathrm{~g} / \mathrm{lm}$ Serum,

- vorhandene Impftiter, z. B. gegen Tetanustoxin (ist dieser niedrig oder negativ, wird ein Booster unter IT mit Verlaufstiterkontrolle empfohlen).

\section{Allgemeine und krankheits- spezifische Impfungen}

Bei der Reiseimpfberatung sollten neben den klassischen Reiseimpfungen bei Patienten mit RE ggf. auch alle bisher nicht erfolgten allgemein oder krankheitsbedingt empfohlenen Impfungen unter Berücksichtigung möglicher Kontraindika- tionen nachgeholt werden [4] (• Tab. 2). Bei sexuellem Risikoverhalten und bei Jugendlichen sollte die Impfung gegen $\mathrm{He}$ patitis B und humane Papillomviren besprochen werden, und bei unzureichender Immunisierung sollte diese empfohlen bzw. nachgeholt werden.

\section{Hepatitis B}

Hepatitis B wird durch das Hepatitis-BVirus ausgelöst. Die Übertragung erfolgt v. a. durch Intimkontakte, mukokutane Kontakte mit infektiösem Material, kontaminierte Bluttransfusionen und perinatal. Die Hepatitis-B-Impfung sollte entsprechend der STIKO Empfehlung erfolgen [27]. Bei Risikopersonen sollte 4 bis 8 Wochen nach der Grundimmunisierung eine Anti-HBs(Hepatitis B-Surface)-AK-Kontrolle erfolgen [29]. Abhängig vom serologischen Erfolg kann eine vierte Impfdosis indiziert bzw. je nach Ansprechen anschließend ein Impfstoffwechsel mit höherer Antigendosis oder zusätzlichem Adjuvans nötig sein [9].

\section{Humanpathogene Papillomaviren}

Das Risiko für HPV(humanpathogene Papillomaviren)-Infektionen ist bei Immunsupprimierten höher als bei $\mathrm{Ge}$ sunden, besonders auch beim systemischen Lupus erythematodes [29, 30]. Die STIKO empfiehlt 9- bis 14-jährige Jungen und Mädchen mit 2 Impfdosen im Abstand von $\geq 5$ Monaten zu impfen [27]. Nachholimpfungen für ältere Personen beinhalten eine dritte Impfdosis [27]. Da die AK-Spiegel bei Immunsup- 
Z Rheumatol 2020 · 79:865-872 https://doi.org/10.1007/s00393-020-00852-w

(c) Der/die Autor(en) 2020

T. Welzel · A. Wörner $\cdot$ U. Heininger

Reiseimpfungen bei rheumatischen Erkrankungen. Besonderheiten bei Kindern und Erwachsenen

\section{Zusammenfassung}

Kinder und Erwachsene mit rheumatischen Erkrankungen (RE) haben nicht nur durch ihre Grunderkrankung, sondern auch durch die vielfach notwendige immunsuppressive Therapie (IT) ein erhöhtes Risiko, an bestimmten Infektionen zu erkranken. Durch die IT hat sich die Lebensqualität bei vielen Patienten mit RE verbessert, sodass ihr internationales Reiseverhalten dem gesunder Reisenden ähnelt. Eine Untersuchung deutet an, dass Patienten mit Immunsuppression oftmals unzureichend auf Reisen vorbereitet sind und ihr Impfschutz schlechter als bei Immungesunden ist. Da auch während und nach Reisen das Erkrankungsrisiko für allgemeine und reisespezifische Infektionen bei Patienten mit Immunsuppression erhöht ist, sind reisemedizinische Beratungen bei Patienten mit RE wichtig. Hier können ein Reiserücktritt oder Reisemodifikationen und spezifischer Schutz inklusive Reiseimpfungen besprochen werden. Zu den gängigen Indikationsimpfungen bei Reisen zählen Impfungen gegen Hepatitis A, Typhus, Tollwut, Meningokokken, FSME (FrühsommerMeningoenzephalitis), saisonale Influenza, Japanische Enzephalitis, Cholera, Poliomyelitis und Gelbfieber. Bei Patienten mit RE ergibt sich die Impfindikation dabei aus der möglichen Exposition gegenüber impfpräventablen Infektionen, dem individuellen Reiseverhalten, der Schwere der möglichen Infektion unter Abwägung der Risiken, die mit der Reiseimpfung assoziiert sind. Dafür müssen auch der allgemeine Gesundheitszustand, die Aktivität und Schwere der RE und der Grad der IT beachtet werden. Allgemein gilt für Patienten mit RE und IT, dass auch bei Reiseimpfungen Lebendimpfstoffe kontraindiziert sind, während Totimpfstoffe verabreicht werden können. Da eine reduzierte Impfantwort möglich ist, können Antikörpermessungen nach der Impfserie und spezifische Impfschemata oder zusätzliche Booster bei Patienten mit RE indiziert sein.

\section{Schlüsselwörter}

Immunsuppression - Lebendimpfstoff . Totimpfstoff · Infektion · Impfschutz

\section{Travel vaccinations in rheumatic diseases. Specific considerations in children and adults}

\section{Abstract}

Children and adults with rheumatic diseases (RD) have a higher risk to contract infections due to the underlying disease and the frequently necessary immunosuppressive treatment (IT). The quality of life of the majority of patients with RD has remarkably improved due to IT-related reduction of inflammation. Therefore, RD patients usually have an international travel behavior similar to healthy individuals. An investigation indicated that patients with RD and IT have lower travel vaccination rates and are often less well-prepared for their trip in comparison to healthy travelers, even when visiting high risk destinations. As the risk for general and travel-acquired infections is increased for patients with RD with and without IT, pretravel consultations are important. These pretravel consultations should include recommendations addressing travel cancellation, travel modification and travel vaccinations depending on the patient's risk. Travel vaccinations include vaccinations against hepatitis $A$, typhoid fever, rabies, cholera, meningococcal diseases, tickbone encephalitis, Japanese encephalitis, seasonal influenza, poliomyelitis and yellow fever. In patients with RD the indications for vaccination depend on the exposure risks, disease severity, individual travel behavior, and possible complications associated with vaccination. In the further evaluation process it is crucial to include the general health condition of the patient, the underlying RD (type and activity), duration and intensity of the IT. In general, live-attenuated vaccines are contraindicated under IT. In contrast, inactivated vaccines may be administered although reduced immunogenicity with the need for antibody measurement, special vaccine schedules or additional booster vaccinations should be considered under IT.

\section{Keywords}

Immunosuppression - Live vaccine . Inactivated vaccine - Infection - Vaccination protection primierten im Vergleich zu Gesunden reduziert sein können, können diese altersunabhängig von einem 3-DosenImpfschema profitieren [29]. Die vollständige Impfserie sollte vor dem ersten Sexualkontakt abgeschlossen sein [27].

\section{Reiseimpfungen}

Bei den Reiseimpfungen wird unterschieden zwischen [8, 18]:

- Pflichtimpfungen, die zur Einreise in einzelnen Ländern vorgeschrieben sind (z. B. Gelbfieberimpfung),
- Standardimpfungen, die nach dem STIKO-Impfkalender vorliegen sollten, und

- Indikationsimpfungen, die entsprechend dem Reiserisiko angezeigt sind.

Zu den Reiseimpfungen (• Tab. 2) zählen beispielsweise Hepatitis A, Typhus, Tollwut, Meningokokken, FSME (Frühsommer-Meningoenzephalitis), saisonale Influenza, Japanische Enzephalitis, Cholera und Gelbfieber [8]. Die Impfung gegen Gelbfieber, Masern-Mumps-
Röteln, Varizellen sowie die orale Typhusimpfung erfolgen mit Lebendimpfstoffen. Die Gelbfieberimpfviren haben eine hohe Replikationskapazität, der orale Typhusimpfstoff hat eine geringe [4]. Allgemein sollten keine Reiseimpfungen mit Lebendimpfstoffen bei Patienten mit RE und IT erfolgen, allerdings kann nach individueller Risikoabwägung unter entsprechenden Vorsichtsmaßnahmen eine Impfindikation gestellt werden [4]. Lebendimpfungen können mit gewisser Latenz nach IT-Stopp erfolgen, dabei variieren die Zeitintervalle je nach IT 
Tab. 1 Orientierende Einordnung des Schweregrades der immunsuppressiven Therapie. (Mod. nach [29])

\begin{tabular}{|c|c|}
\hline $\begin{array}{l}\text { Schweregrad der immun- } \\
\text { suppressiven Wirkung }\end{array}$ & Therapie \\
\hline \multirow[t]{3}{*}{ Keine oder gering } & Hydroxychloroquin \\
\hline & Sulfasalazin \\
\hline & Mesalazin \\
\hline \multirow[t]{7}{*}{ Gering } & $\begin{array}{l}\text { Niedrigdosierte systemische Glukokortikoide } \\
\text { - Erw.: }<10 \mathrm{mg} \text { Prednisolonäquivalent/Tag } \\
\text { - Kinder: }<0.2 \mathrm{mg} / \mathrm{kg} / \mathrm{Tag} \text { Prednisolonäquivalent } \\
\text { - Kurzzeittherapie ( }<2 \text { Wochen) }\end{array}$ \\
\hline & $\begin{array}{l}\text { Nicht systemische Glukokortikoide } \\
\text { - Topisch (dermal, inhalativ, konjunktival, nasal) } \\
\text { - Intraartikulär }\end{array}$ \\
\hline & $\begin{array}{l}\text { Niedrigdosierte Basistherapeutika } \\
\text { MTX } \\
\text { - Erw.: } \leq 0.4 \mathrm{mg} / \mathrm{kg} / \text { Woche oder } \leq 20 \mathrm{mg} / \text { Woche } \\
\text { - Kinder } \leq 15 \mathrm{mg} / \mathrm{m}^{2} / \text { Woche }\end{array}$ \\
\hline & $\begin{array}{l}\text { Ciclosporin }(\leq 2.5 \mathrm{mg} / \mathrm{kg} / \mathrm{Tag}) \\
\text { Leflunomid } \\
\text { - Erw.: } \leq 20 \mathrm{mg} / \mathrm{Tag} \\
\text { - Kinder } \leq 0.5 \mathrm{mg} / \mathrm{kg} / \mathrm{Tag}\end{array}$ \\
\hline & $\begin{array}{l}\text { Mycophenolat-Mofetil } \\
\text { - Erw.: } \leq 2000 \mathrm{mg} / \mathrm{Tag} \\
\text { - Kinder } \leq 1200 \mathrm{mg} / \mathrm{m}^{2} / \mathrm{Tag}\end{array}$ \\
\hline & $\begin{array}{l}\text { Tofacitinib } \\
\text { - Erw.: } \leq 5-10 \mathrm{mg} / \mathrm{Tag}\end{array}$ \\
\hline & $\begin{array}{l}\text { Einige niedrigdosierte Biologika, } z \text {. B. } \\
\text { Infliximab ( } \leq 3 \mathrm{mg} / \mathrm{kg} / 8 \text { Wochen) }\end{array}$ \\
\hline \multirow[t]{5}{*}{ Schwer } & $\begin{array}{l}\text { Hochdosierte systemische Glukokortikoide } \\
\text { - Dosierung oberhalb der oben genannten Grenzwerte } \\
\text { - Therapiedauer } \geq 2 \text { Wochen } \\
\text { - Intravenöse Stosstherapie mit hohen Dosen (z. B. } 20 \mathrm{mg} / \mathrm{kg} / \mathrm{Tag} \\
\text { Prednisolonäquivalent über mehrere Tage monatlich wieder- } \\
\text { holt) }\end{array}$ \\
\hline & $\begin{array}{l}\text { Hochdosierte Basistherapeutika } \\
\text { - Dosierung oberhalb der oben genannten Grenzwerte }\end{array}$ \\
\hline & Azathioprin \\
\hline & $\begin{array}{l}\text { Biologika mit schwerer Immunsuppressiver Wirkung, z. B. } \\
\text { - Infliximab ( } \geq 5 \mathrm{mg} / \mathrm{kg} / 4 \text { Wochen bzw. } \geq 7 \mathrm{mg} / \mathrm{kg} / 8 \text { Wochen) } \\
\text { - Abatacept } \\
\text { - Rituximab }\end{array}$ \\
\hline & Kombinationen von Immunsuppressiva \\
\hline
\end{tabular}

und Impfung $[4,10,13]$. Reiseimpfungen mit Totimpfstoff gelten als sicher, allerdings kann der Impfschutz bei RE mit und ohne IT reduziert sein [4, 19, 29].

können [23]. Die Impfung kann zusätzlich bei Aufenthalt in Infektionsgebieten speziell unter mangelhaften Hygienebedingungen bei aktuellen Ausbrüchen z. B. in Flüchtlingslagern oder bei Naturkatastrophen indiziert sein [27]. Der orale Totimpfstoff kann nach Fachinformation bei Patienten mit Immunsuppression verabreicht und sollte je nach Reiseland, Reisedauer und erwarteter Exposition empfohlen werden [19, 29], allerdings sollte berücksichtigt werden, dass die Wirksamkeit bei vorübergehend Exponierten unbekannt zu sein scheint und auch kein Schutz gegen Serovar O139 besteht [23]. Die impfinduzierten antitoxischen AK im Darm können darüber hinaus einen partiellen Schutz gegen das „Cholera-like-Toxin“ der enterotoxischen Escherichia coli bieten [8].

\section{Frühsommer-Meningoenzephalitis}

FSME wird durch Flaviviren ausgelöst und überwiegend durch die Schildzecke Ixodes ricinus übertragen. Die FSMEImpfung wird allen Personen empfohlen, die in Risikogebieten zeckenexponiert sind [27]. Die Impfung sollte auch bei Patienten mit RE mit und ohne IT entsprechend den STIKO-Empfehlungen erfolgen [29]. Bei Patienten mit IT kann die Impfantwort eingeschränkt sein [15].

\section{Gelbfieber}

Gelbfieber wird durch ein Flavivirus verursacht. Der Hauptvektor ist die tagesaktive Mücke Aedes aegypti. Die Gelbfieberimpfung ist bei Immunsuppression kontraindiziert [4, 11, 19, 25, 29]. Personen mit schwerer Immunsuppression sollte von Reisen in Gelbfieberendemiegebiete abgeraten werden $[19,25,29]$.

\section{Spezifische Informationen zu einzelnen Reiseimpfungen}

\section{Cholera}

Cholera wird durch Aufnahme von kontaminierten Nahrungsmitteln/Trinkwasser durch das Bakterium Vibrio cholerae ausgelöst. Maßnahmen zur Nahrungs-und Trinkwasserhygiene sollten beachtet werden, da diese bereits alleine hocheffektiv eine Choleraübertragung verhindern

\section{》) Die Gelbfieberimpfung ist bei Immunsuppression kontraindiziert}

Für die Einreise in bestimmte Länder ist der Nachweis einer Impfung erforderlich. Bei medizinischer Kontraindikation und Reisenotwendigkeit kann ein „exemption certificate“ ausgestellt werden, das von einer Impfung entbindet 
Tab. 2 Reiseimpfungen bei Patienten mit rheumatischen Erkrankungen. (Mod. nach [23, 24, 27, 29])

\begin{tabular}{|c|c|c|c|}
\hline Impfung gegen & Lebendimpfstoff & Totimpfstoff & Mindestalter und Kommentare \\
\hline Cholera & - & $x$ & DUKORAL ${ }^{\circledR}$ (Valneva Sweden AB, Schweden): $2 \mathrm{~J}$ \\
\hline $\begin{array}{l}\text { Frühsommer-Meningo- } \\
\text { enzephalitis }\end{array}$ & - & $\mathrm{x}$ & $\begin{array}{l}\text { Encepur }{ }^{\circledR} \text { Kinder (GlaxoSmithKline, Vereinigtes Königreich)/FSME-Immun }{ }^{\circledR} \\
0,25 \text { ml Junior (Pfizer Pharma, Vereinigte Staaten): } 1 \mathrm{~J} . \\
<3 \text { J. Fieberreaktionen häufig, individuelle Indikationsstellung }\end{array}$ \\
\hline Gelbfieber & $x$ & - & $\begin{array}{l}\text { STAMARIL }^{\circledR} \text { (Sanofi, Frankreich): } 9 \text { (6) Mo, KI: IT } \\
\text { Zugelassen ab } 6 \text { Mo., allerdings erhöhtes Enzephalitisrisiko zwischen } 6 \text { und } \\
9 \text { Mo, daher strenge Risiko-Nutzen-Evaluation }\end{array}$ \\
\hline Hepatitis A & - & $x$ & $\begin{array}{l}\text { Havrix }{ }^{\circledR} 720 \text { Kinder (GlaxoSmithKline, Vereinigtes Königreich)/VAQTA }{ }^{\circledR} \text { Kinder } \\
\text { (MSD Merck Sharp \& Dohme, Vereinigte Staaten): } 1 \mathrm{~J} . \\
\text { Havrix } 1440^{\circledR} \text { (GlaxoSmithKline, Vereinigtes Königreich): } 15 \mathrm{~J} . \\
\text { VAQTA }^{\circledR} \text { (MSD Merck Sharp \& Dohme, Vereinigte Staaten): } 18 \mathrm{~J} .\end{array}$ \\
\hline Hepatitis A+B & - & $x$ & $\begin{array}{l}\text { Twinrix }{ }^{\circledR} \text { Kinder (GlaxoSmithKline, Vereinigtes Königreich): } 1 \mathrm{J.} \\
\text { Twinrix }^{\circledR} \text { (GlaxoSmithKline, Vereinigtes Königreich): } 16 \mathrm{~J} .\end{array}$ \\
\hline Japanische Enzephalitis & - & $x$ & IXIARO ${ }^{\circledR}$ (diverse Hersteller): 2 Mo. \\
\hline Meningokokken B & - & $\mathrm{x}$ & $\begin{array}{l}\text { Bexsero }^{\circledR} \text { (GlaxoSmithKline, Vereinigtes Königreich): } 2 \text { Mo. } \\
\text { Trumenba }{ }^{\circledR} \text { (Pfizer Pharma, Vereinigte Staaten): } 10 \mathrm{~J} .\end{array}$ \\
\hline $\begin{array}{l}\text { Meningokokken } \\
\text { ACWY-Konjugat }\end{array}$ & - & $x$ & $\begin{array}{l}\text { Menveo }^{\circledR}(\text { GlaxoSmithKline, Vereinigtes Königreich): } 2 \mathrm{~J} . \\
\text { Nimenrix }^{\circledR} \text { (Pfizer Pharma, Vereinigte Staaten): } 6 \text { Wochen }\end{array}$ \\
\hline Poliomyelitis & - & $x$ & $\begin{array}{l}\text { IPV Merieux }{ }^{\circledR} \text { (Sanofi, Frankreich) /Kombinationsimpfung (diverse Hersteller): } \\
\text { Grundimmunisierung gemäß STIKO ab dem Alter von } 2 \text { Mo. (idealerweise als } \\
\text { Kombinationsimpfung), Reiseindikation beachten }\end{array}$ \\
\hline Tollwut & - & $x$ & $\begin{array}{l}\text { Rabipur }^{\circledR} \text { (GlaxoSmithKline, Vereinigtes Königreich)/Tollwutimpfstoff (HDC) } \\
\text { inaktiviert }{ }^{\circledR} \text { (Sanofi, Frankreich): ab Geburt }\end{array}$ \\
\hline Typhus & 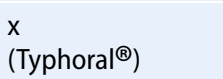 & $\begin{array}{l}\text { x } \\
\left(\text { Typhim Vi }{ }^{\circledR}\right)\end{array}$ & $\begin{array}{l}\text { Typhim Vi } \mathrm{P}^{\circledR} \text { (Sanofi, Frankreich): } 2 \mathrm{~J} \text {. } \\
\text { Typhoral }{ }^{\circledR} \text { L Kapseln (Pharma K Medical GmbH; Deutschland): } 5 \mathrm{J.} \text { KI: IT }\end{array}$ \\
\hline \multirow{2}{*}{\multicolumn{4}{|c|}{$\begin{array}{l}\text { Die STIKO-Standardimpfungen [ 27] sowie krankheitsspezifische Impf } \\
\text { gemäß aktuell sein: }\end{array}$}} \\
\hline & & & \\
\hline \multicolumn{4}{|c|}{ Hepatitis B } \\
\hline \multicolumn{4}{|c|}{ Humanpathogene Papillomaviren } \\
\hline \multicolumn{4}{|l|}{ Pneumokokken } \\
\hline $\begin{array}{l}\text { MMR } \\
\text { - Idealerweise vor IT (2 D } \\
\text { - KI bei schwerer Immun } \\
\text { - Niedrigdosierte Glukok } \\
\text { - Verabreichung laut Fac } \\
\text { gung möglich }\end{array}$ & $\begin{array}{l}\text { ienz/IT } \\
\text { oide sind keine KI } \\
\text { rmationen und Exp }\end{array}$ & rtenkonsens im & zelfall bei geringer Immunsuppression nach individueller Risiko-Nutzenabwä- \\
\hline \multicolumn{4}{|c|}{$\begin{array}{l}\text { - Verabreichung laut Fachinformationen nach individueller Risiko-Nutzenabwägung in Abhängigkeit von der Immunsuppression unter Therapie/in } \\
\text { Therapiepausen nach immunologischer Vordiagnostik möglich, wenn ein besonderes Gesundheitsrisiko vorliegt und die Gesamtlymphozytenzahl } \\
\text { bei Erwachsenen mindestens 1200/mm } \mathrm{mm}^{3} \text { Blut beträgt (bei Kindern altersabhängige Referenzwerte) und/oder kein anderer Hinweis auf unzurei- } \\
\text { chende zelluläre Immunität besteht }\end{array}$} \\
\hline \multicolumn{4}{|c|}{ Influenza (KI: nasaler Lebendimpfstoff bei IT) } \\
\hline$I T \mathrm{i}$ & ahro $K I$ & & : \\
\hline
\end{tabular}

$[19,24,29]$. Die Persistenz von neutralisierenden AK scheint bei Patienten, die vor IT gegen Gelbfieber geimpft worden sind, nicht beeinflusst [6]. Allerdings ist für eine Langzeitimmunität eine zweite Impfdosis empfohlen [6].

\section{Hepatitis A}

Hepatitis A wird durch das HepatitisA-Virus fäkal-oral übertragen und ist eine häufige Infektionskrankheit bei Reisenden in Risikogebiete. Die Impfung wird allen, auch Immunsupprimierten, ab dem Alter von 1 Jahr bei Reisen in Regionen mit hoher Hepatitis-A-
Inzidenz empfohlen [27, 29]. Studien zeigen bei Patienten mit IT im Vergleich $\mathrm{zu}$ Immunkompetenten, insbesondere unter Tumor-Nekrose-Faktor(TNF)Inhibitoren, eine unzureichende Seroprotektion nach erster Impfdosis [1, 28]. Eine zusätzliche Impfdosis parallel zur ersten oder 4 Wochen nach erster Dosis kann bei IT den Impferfolg verbessern 
[22]. Eine dritte Dosis sollte nach 6 bis 12 Monaten verabreicht werden, um einen Langzeitschutz sicherzustellen [29].

\section{Japanische Enzephalitis (JE)}

Die JE ist eine Flavivirus-Infektion, die durch die nachtaktive Culexmücke in Teilen von Asien und der westpazifischen Region übertragen wird [24]. Sie ist eine der häufigsten Ursachen weltweit für virale Enzephalitiden und geht mit einer Letalität von etwa $30 \%$ sowie bei Überlebenden mit hohen Raten an Residualschäden einher [18, 24]. In Deutschland ist ein Totimpfstoff ab 2 Monaten zugelassen und wird in 2 Dosen im Abstand von 4 Wochen verabreicht. Im Alter von 2 Monaten bis < 3 Jahre wird die Hälfte des Impfstoffs verabreicht, ab 3 Jahren wird die volle Dosis gegeben [24]. Bei Reisen in Endemiegebiete scheint für Patienten mit Immunsuppression, insbesondere unter Therapie mit TNF-Inhibitoren das Risiko für die JE erhöht, sodass die Impfung bei dieser Personengruppe besonders indiziert zu sein scheint $[5,29]$.

\section{Meningokokken (B, ACWY)}

Die Meningokokkenmeningitis mit/ ohne Sepsis wird durch invasive Stämme von Neisseria meningitidis hervorgerufen und kann lebensbedrohlich verlaufen. Bei der Meningokokkenimpfung muss die Epidemiologie berücksichtigt werden. Die in Deutschland verfügbaren quadrivalenten Meningokokkenkonjugatimpfstoffe schützen vor Serogruppen ACWY, aber nicht vor Serogruppe B. Es sind 2 Impfstoffe gegen B-Meningokokken in Deutschland zugelassen [24]. Eine Impfung gegen die Serogruppe C wird in den STIKO-Standardimpfungen bis 18 Jahre empfohlen [27]. Die quadrivalente Meningokokkenimpfung sollte allen Reisenden in Endemiegebiete, z. B. mitZiel afrikanischer „Meningitisgürtel“, empfohlen werden [18, 24, 27]. Der Meningitisgürtel zieht sich von Senegal und Gambia bis nach Äthiopien [24]. Auch können Impfindikationen bei Reisen in (sub)tropische Regionen mit niedrigem sozioökonomischem Status und engem Kontakt zu Einheimischen, bei Langzeit- schüleraustausch oder Studienaufenthalt im Ausland bestehen [24]. Bei Patienten mit Immunsuppression kann die Impfantwort reduziert sein [29]. Es gibt daher z.B. in der Schweiz die Empfehlung, Patienten mit erhöhtem Risiko für invasive Meningokokkenerkrankungen im Alter von 2 bis 11 Monaten mit 4 Dosen (Zeitpunkt 2, 3, 4 und 12 Monate) und im Alter $\geq 12$ Jahren mit 2 Dosen des quadrivalenten Impfstoffs im Abstand von 4 bis 8 Wochen zu impfen [2]. Bei fortbestehendem Infektionsrisiko sollten alle 5 Jahre Booster erfolgen [2].

\section{Poliomyelitis}

Die Poliomyelitis wird durch fäkalorale Infektion mit dem Poliovirus verursacht. Eine Grundimmunisierung einschließlich Auffrischimpfung gegen Poliomyelitis wird in den STIKO-Standardimpfungen empfohlen [27]. Sowohl beim Kombinationsimpfstoff (Diphtherie, Pertussis, Tetanus, Polio) wie auch bei dem inaktivierten Poliovakzin (IPV) handelt es ich um Totimpfstoffe, sodass auch Patienten mit IT geimpft werden können. Eine IPV-Auffrischimpfung wird bei Reisen in Regionen mit Infektionsrisiko empfohlen, wenn die letzte Impfung bei abgeschlossener Grundimmunisierung $>10$ Jahre zurückliegt [27]. Neben formalen Impfindikationen (Länderbestimmungen beachten) hat die WHO (Weltgesundheitsorganisation) auch für bestimmte Länder verschärfte temporäre Impfempfehlungen ausgesprochen, sodass kürzere Impfabstände gelten können (Informationen des Auswärtigen Amts beachten) [8, 27]. Bei Personen ohne Grundimmunisierung sollten vor Reisebeginn mindestens 2 IPV-Impfdosen im 4-Wochen-Abstand verabreicht werden [27]. Bei fehlenden oder nicht dokumentierten Impfungen der Grundimmunisierung sollten diese nachgeholt werden [27].

\section{Tollwut}

Der Tollwuterreger ist das Rabiesvirus, das bei einem Biss von infizierten Säugetieren übertragen werden kann. Die klassische Form der Tollwut verläuft letal. Die Tollwutimpfung ist bei Reisen in Regionen mit erhöhter Tollwutgefährdung empfohlen [27]. In Deutschland sind 2 Totimpfstoffe auf Basis inaktivierter Tollwutviren zugelassen [24]. Die präexpositionelle Tollwutimpfung (HDC[human diploid cell]- oder PCEC[purified chick embryo cell]-Impfstoff) wird nach dem in der Fachinformation genannten Impfschema (3 Impfstoffdosen Tag 0, 7, 28) empfohlen [9]. Ein Schnellimpfschema („off-label“) wird nicht empfohlen [9]. Für immundefiziente Personen ist eine AK-Bestimmung 2 bis 4 Wochen nach vollständiger Grundimmunisierung mit erneuter Impfung bei einem AK-Spiegel $<0,5 \mathrm{IE} / \mathrm{ml}$ empfohlen $[8,9]$. Dies Vorgehen empfehlen wir auch bei Patienten mit RE mit und ohne IT. Bei länger notwendigem Impfschutz kann durch AK-Bestimmung abgeschätzt werden, ob eine Auffrischimpfung notwendig ist und empfohlen werden sollte. Auch kann man sich an den Anwendungshinweisen für Reisende mit Immundefizienz orientieren. Hier wird eine Auffrischung $1 \mathrm{Jahr}$ nach erster Impfung und dann alle 5 Jahre mit je einer Impfdosis empfohlen [9]. Kommt es bei Ungeimpften zu Kontakt mit tollwutverdächtigen Tieren (Biss-/ Kratzwunden, Speichelkontakt mit offenen Hautstellen/Schleimhäuten), muss umgehend eine Postexpositionsprophylaxe (PEP) durchgeführt werden [18].

\section{》) Bei Ungeimpften mit Kontakt zu tollwutverdächtigen Tieren muss umgehend eine PEP durchgeführt werden}

Bei Patienten mit Immunsuppression sollte dabei bei Exposition ab Grad II eine PEP mit 5 Impfstoffdosen nach dem Essen-Schema an Tag 0, 3, 7, 14, 28 eingeleitet werden, auch wenn eine präexpositionelle Impfserie durchgeführt wurde $[9,19]$. Eine simultane Gabe von Immunglobulinen sollte erfolgen und kann bis zu 7 Tage nach Beginn der PEP nachgeholt werden $[9,19]$. Die Fachinformation sollte beachtet werden. Eine Titerbestimmung sollte 7 bis 14 Tage nach der letzten Impfung im Rahmen der PEP erfolgen $[9,19]$. 


\section{Typhus}

Typhus abdominalis wird durch Salmonella typhi über infizierte Nahrungsmittel, seltener durch Schmierinfektion ausgelöst. Die Impfung sollte bei Reisen nach Südasien und in andere Endemiegebiete, bei Langzeitaufenthalten mit niedrigen Hygienestandards und bei Naturkatastrophen erfolgen [24]. Je nach Reiseland, Reisedauer und Expositionsrisiko sollten auch Patienten mit RE mit und ohne IT mit dem inaktivierten Typhusimpfstoff geimpft werden $[19,29]$. Bei Risikopatienten sollte die Impfindikation großzügiger als bei Immunkompetenten gestellt werden [19].

\section{Fazit für die Praxis}

\section{- Patienten mit rheumatischen Erkran-} kungen sollten vor internationalen Reisen eine reisemedizinische Beratung erhalten, in der unter Beachtung des individuellen Infektionsrisikos Reiserücktritt, Reisemodifikationen, Schutzmaßnahmen und Reiseimpfungen besprochen werden.

- Die Indikation für eine Reiseimpfung ergibt sich aus reisespezifischen Faktoren, dem allgemeinen Gesundheitszustand, der Art und Aktivität der Grundkrankheit und Dauer, Dosis und Art der immunsuppressiven Therapie.

- Fachinformationen der Impfstoffe und immunsuppressiven Medikamente müssen beachtet werden; auch sind ggf. individuelle RisikoNutzen-Abwägungen wichtig.

- Reiseimpfungen mit Totimpfstoffen können bedenkenlos erfolgen, wohingegen Lebendimpfstoffe (z. B. Gelbfieberimpfung) allgemein unter immunsuppressiver Therapie kontraindiziert sind.

- Der Impferfolg, beeinflusst durch Grunderkrankung und Therapie, kann reduziert sein, daher sollten Antikörperbestimmungen 4 bis 8 Wochen nach abgeschlossener Impfserie erwogen werden.

- Zusätzliche Impfdosen können für den optimierten Impfschutz nötig sein (z. B. Hepatitis A).

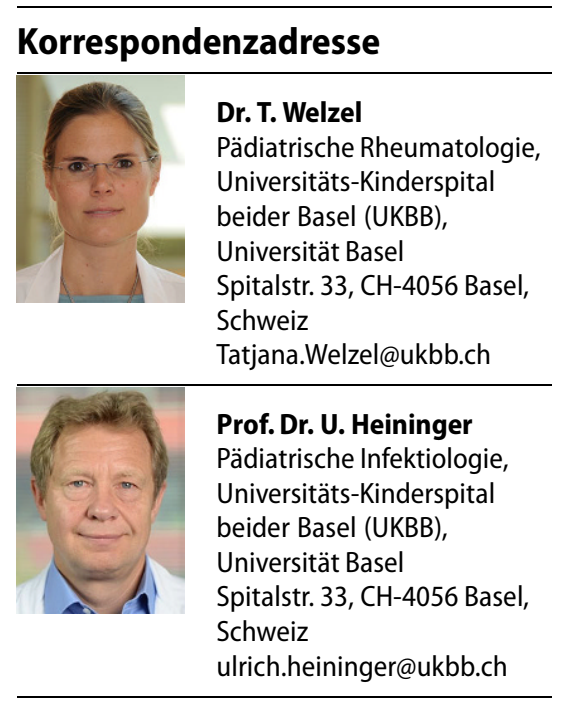

Funding. Open access funding provided by University of Basel

\section{Einhaltung ethischer Richtlinien}

Interessenkonflikt. U. Heininger hat Honorare für Vorträge zu Impfthemen ohne Produktbezug von MSD, Pfizer, und Sanofi erhalten. T. Welzel und A. Wörner erklären, keine finanziellen oder persönlichen Beziehungen zu Dritten zu haben, deren Interesse den Beitragsinhalt positiv oder negativ beeinflusst haben könnte. Es besteht kein Interessenkonflikt.

Dieser Beitrag basiert auf bereits publizierter Literatur. Der Beitrag enthält keine neuen eigenen Studienergebnisse aus Forschung am Menschen oder an Tieren.

Open Access Dieser Artikel wird unter der Creative Commons Namensnennung 4.0 International Lizenz veröffentlicht, welche die Nutzung, Vervielfältigung, Bearbeitung, Verbreitung und Wiedergabe in jeglichem Medium und Format erlaubt, sofern Sie den/die ursprünglichen Autor(en) und die Quelle ordnungsgemäß nennen, einen Link zur Creative Commons Lizenz beifügen und angeben, ob Änderungen vorgenommen wurden.

Die in diesem Artikel enthaltenen Bilder und sonstiges Drittmaterial unterliegen ebenfalls der genannten Creative Commons Lizenz, sofern sich aus der Abbildungslegende nichts anderes ergibt. Sofern das betreffende Material nicht unter der genannten Creative Commons Lizenz steht und die betreffende Handlung nicht nach gesetzlichen Vorschriften erlaubt ist, ist für die oben aufgeführten Weiterverwendungen des Materials die Einwilligung des jeweiligen Rechteinhabers einzuholen.

Weitere Details zur Lizenz entnehmen Sie bitte der Lizenzinformation auf http://creativecommons.org/ licenses/by/4.0/deed.de.

\section{Literatur}

1. Askling $H H$, Rombo L, Van Vollenhoven R et al (2014) Hepatitis A vaccine for immunosuppressed patients with rheumatoid arthritis: a prospective, open-label, multi-centre study. Travel Med Infect Dis 12:134-142

2. Bundesamt für Gesundheit, Eidgenössische Kommission für Impffragen (2020) Schweizerischer Impfplan 2020. Richtlinien und Empfehlungen. Bundesamt für Gesundheit, Bern

3. Bialy C, Horne K, Dendle Cetal (2015) International travel in theimmunocompromised patient:acrosssectional survey of travel advice in 254 consecutive patients. Intern Med J 45:618-623

4. Buhler S, Eperon G, Ribi C et al (2015) Vaccination recommendations for adult patients with autoimmune inflammatory rheumatic diseases. Swiss Med Wkly 145:w14159

5. Burchard GD, Caumes E, Connor BA et al (2009) Expert opinion on vaccination of travelers against Japanese encephalitis. J Travel Med 16:204-216

6. Burkhard J, Ciurea A, Gabay Cetal (2020) Long-term immunogenicity after yellow fever vaccination in immunosuppressed and healthy individuals. Vaccine. https://doi.org/10.1016/j.vaccine.2019. 12.042

7. Dekkiche S, De Valliere S, D'acremont V et al (2016) Travel-related health risks in moderately and severely immunocompromised patients: a casecontrol study. J Travel Med. https://doi.org/10. 1093/jtm/taw001

8. Deutsche Gesellschaft für Tropenmedizin und Internationale Gesundheit (DTG) (2017) Hinweise und Empfehlungen zu Reiseimpfungen, S1-39

9. Ehl S, Bogdan C, Niehues T et al (2018) Impfen bei Immundefizienz Anwendungshinweise zu den von der Ständigen Impfkommission empfohlenen Impfungen. (II) Impfen bei 1. Primären Immundefekterkrankungen und 2. HIV-Infektion. Bundesgesundheitsblatt Gesundheitsforschung Gesundheitsschutz 61:1034-1051

10. Freedman DO, Chen LH (2019) Vaccines for international travel. Mayo Clin Proc 94:2314-2339

11. Furer V, Rondaan C, Heijstek MW et al (2020) 2019 update of EULAR recommendations for vaccination in adult patients with autoimmune inflammatory rheumatic diseases. Ann Rheum Dis 79:39-52

12. Groot N, Heijstek MW, Wulffraat NM (2015) Vaccinations in paediatric rheumatology: an update on current developments. Curr Rheumatol Rep 17:46

13. Hall V, Johnson D, Torresi J (2018) Travel and biologic therapy: travel-related infection risk, vaccine response and recommendations. J Travel Med. https://doi.org/10.1093/jtm/tay018

14. Heijstek MW, Ott De Bruin LM, Bijl M et al (2011) EULAR recommendations for vaccination in paediatric patients with rheumatic diseases. Ann Rheum Dis 70:1704-1712

15. Hertzell KB, Pauksens K, Rombo L et al (2016) Tick-borne encephalitis (TBE) vaccine to medically immunosuppressed patients with rheumatoid arthritis: A prospective, open-label, multi-centre study. Vaccine 34:650-655

16. Horneff G (2015) Biologic-associated infections in pediatric rheumatology. Curr Rheumatol Rep 17:66

17. Jaeger VK, Ruegg R, Steffen R et al (2015) Travelers with immune-mediated inflammatory diseases: are they different? J Travel Med 22:161-167

18. Jelinek T (2018) What is verified for travel vaccinations? Internist 59:1255-1267

19. Kling K, Wichmann O, Burchard G (2020) Travel vaccinations for certain groups of persons. Bundesgesundheitsblatt Gesundheitsforschung Gesundheitsschutz 63:85-92 
20. Lahiri M, Dixon WG (2015) Risk of infection with biologic antirheumatic therapies in patients with rheumatoid arthritis. Best practice \& research. Clin Rheumatol 29:290-305

21. Niehues T, Bogdan C, Hecht J et al (2017) Impfen bei Immundefizienz Anwendungshinweise zu den von der Ständigen Impfkommission empfohlenen Impfungen (I) Grundlagenpapier Bundesgesundheitsblatt Gesundheitsforschung Gesundheitsschutz 60:674-684

22. Rosdahl A, Herzog C, Frosner G et al (2018) An extra priming dose of hepatitis $A$ vaccine to adult patients with rheumatoid arthritis and drug induced immunosuppression-A prospective, open-label, multi-center study. Travel Med Infect Dis 21:43-50

23. Rothe C, Alberer M, Bühler S et al (2019) Reiseimpfungen-Hinweise und Empfehlungen des Ständigen Ausschusses der Reisemedizin (StAR) der DTG. Flug Reisemed. https://doi.org/10. 1055/a-0852-2732

24. Rothe C, Boecken G, Ständiger Ausschuss Reisemedizin (StAR) der Deutschen Gesellschaft für Tropenmedizin und Globale Gesundheit (DTG) (2020) Travel vaccinations. Bundesgesundheitsblatt63(2020):74-84

25. Rubin LG, Levin MJ, Ljungman P et al (2014) 2013 IDSA clinical practice guideline for vaccination of the immunocompromised host. Clin Infect Dis 58:e44-e100

26. Speth F, Minden K (2015) Impfungen bei Kindern und Jugendlichen mit rheumatischen und muskuloskelettalen Erkrankungen. Arthritis Rheuma 35:44-55

27. Ständige Impfkommission (2019) Empfehlungen der Ständigen Impfkommission (STIKO) am Robert Koch-Institut. Epid Bull 34:313-364

28. Van Den Bijllaardt W, Siers HM, TimmermanKok C et al (2013) Seroprotection after hepatitis a vaccination in patients with drug-induced immunosuppression. JTravel Med 20:278-282

29. Wagner N, Assmus F, Arendt G et al (2019) Impfen bei Immundefizienz Anwendungshinweise zu den von der Ständigen Impfkommission empfohlenen Impfungen. (IV) Impfen bei Autoimmunkrankheiten, bei anderen chronisch-entzündlichen Erkrankungen und unter immunmodulatorischer Therapie. Bundesgesundheitsblatt Gesundheitsforschung Gesundheitsschutz 62:494-515

30. Wiedermann U, Sitte HH, Burgmann Het al (2016) Guidelines for vaccination of immunocompromised individuals. Wien Klin Wochenschr 128(Suppl 4):337-376

\section{Neue Strategie gegen Osteoporose}

Ein internationales Forschungsteam hat einen neuen Ansatzpunkt gefunden, über den man möglicherweise den Knochenabbau bei Osteoporose verringern und die Knochengesundheit erhalten kann.

Weltweit sind hunderte Millionen Menschen von Osteoporose betroffen. Es wird geschätzt, dass eine von drei Frauen und einer von fünf Männern im Alter über 50 an osteoporotischen Knochenbrüchen leidet.

Bei der Osteoporose führt ein zu hoher Knochenabbau zu einer niedrigen Knochenmineraldichte und folglich zu schwachen und bruchgefährdeten Knochen. Wenn die Knochenneubildung nicht in der Lage ist, den Knochenschwund auszugleichen, wird der Knochen geschwächt und ist dann anfälliger für Frakturen.

\section{Gängige Medikamente haben Nachtei-} le

Bei den meisten Osteoporose-Therapien werden Bisphosphonate eingesetzt. Bei längerer Behandlung mit diesen Wirkstoffen entfällt aber der wichtige Materialumsatz im Knochen - das führt am Ende wieder zu einem erhöhten Bruchrisiko und zu anderen unerwünschten Nebenwirkungen. Daher sucht die medizinische Forschung neue Strategien, um die Grenzen der derzeitigen Therapien zu überwinden.

\section{Kleines Protein mobilisiert knochenab-} bauende Zellen

Professor Winkler (Fakultät für Biowissenschaften, National University of Singapore, NUS) und Professor Schartl (Biozentrum, Julius-Maximilians-Universität Würzburg, JMU) haben bei einer genetischen Analyse des Laborfisches Medaka (Oryzias latipes) das kleine Protein CXCL9 als wichtigen Akteur identifiziert, der die Rekrutierung von knochenabbauenden Zellen kontrolliert.

Unter osteoporotischen Bedingungen sammelt sich das Protein in Reservoirs an, die auch Vorläufer von knochenabbauenden Zellen enthalten. Diese Vorläufer produzieren auf ihrer Zelloberfläche den Rezeptor CXCR3. Dieser wird vom Protein CXCL9 aktiviert, die Vorläuferzellen werden dadurch mobilisiert und wandern über weite Strecken zum Knochen, wo sie mit ihrer destruktiven Arbeit beginnen.
Bekannte Hemmstoffe sind hochwirksam

Sowohl von CXCL9 als auch von seinem Rezeptor CXCR3 ist seit langem bekannt, dass sie die Wanderung von Immunzellen zu Entzündungsherden modulieren, zum Beispiel bei Psoriasis und rheumatoider Arthritis. Es gibt mehrere chemische Hemmstoffe, die die Aktivität von CXCR3 blockieren, in klinischen Tests zur Behandlung von Psoriasis bisher aber wenig Erfolg zeigten. Das internationale Forschungsteam zeigt nun, dass genau diese Hemmstoffe hochwirksam die Rekrutierung von knochenabbauenden Zellen blockieren und den Knochen vor der Osteoporose schützen

\section{Fein abgestimmten Therapien schei- nen möglich}

Das Fazit der Professoren Schartl und Winkler: „Unsere Studien eröffnen neue Wege zur Osteoporose-Therapie. Die neue Strategie ermöglicht eine fein abgestimmte Modulation die in die Knochenmatrix rekrutiert werden, anstatt eine generelle Blockade dieser Zellen wie bei herkömmlichen Therapien. Das chenabbau gezielt verhindert werden kann, der normale Knochenumsatz aber erhalten bleibt. Das bietet das Potenzial, erhöhte Frakturrisiken bei Osteoporose-Patienten zu vermeiden und gesunde Knochen für eine verbesserte Lebensqualität zu erhalten.

Originalpublikation

Schartl und Winkler (2020) Cxcl9| and Cxcr3.2 regulate recruitment of osteoclast progenitors to bone matrix in a medaka osteoporosis model. PNAS, 27. Juli 2020, https://doi.org/10.1073/pnas.2006093117 der Anzahl der knochenabbauenden Zellen, hat große Vorteile, da ein übermäßiger Kno- 